\title{
Accuracy of Frozen Section Analysis Versus Specimen Radiography During Breast-Conserving Surgery for Nonpalpable Lesions
}

\author{
Walter P. Weber · Stephan Engelberger · \\ Carsten T. Viehl · Rosanna Zanetti-Dallenbach . \\ Salome Kuster · Stephan Dirnhofer · Daniela Wruk • \\ Daniel Oertli · Walter R. Marti
}

Published online: 4 October 2008

(C) Société Internationale de Chirurgie 2008

\begin{abstract}
Background Whereas specimen radiography (SR) is an established strategy for intraoperative resection margin analysis during breast-conserving surgery for nonpalpable lesions, the use of frozen section analysis (FSA) is still a matter of debate.

Methods A retrospective review was conducted of 115 consecutive operations in which the two objectives sought were the excision of nonpalpable malignant lesions and breast conservation. Breast surgery was performed in the Gynecology and the Surgery Departments at the Basel University Hospital Breast Center. Whereas one department preferably uses SR for intraoperative margin assessments of lesions involving ductal carcinoma in situ
\end{abstract}

Walter P. Weber and Stephan Engelberger have contributed equally to this work.

W. P. Weber · S. Engelberger · C. T. Viehl · D. Oertli

W. R. Marti ( $\square)$

Department of Surgery, University Hospital of Basel,

4031 Basel, Switzerland

e-mail:wrmarti@uhbs.ch

R. Zanetti-Dallenbach

Department of Gynecology, University Hospital of Basel,

4031 Basel, Switzerland

S. Kuster

Department of Anesthesia, University Hospital of Basel,

4031 Basel, Switzerland

S. Dirnhofer

Department of Pathology, University Hospital of Basel,

4031 Basel, Switzerland

D. Wruk

Department of Radiology, University Hospital of Basel,

4031 Basel, Switzerland
(DCIS) or atypical ductal hyperplasia, the other uses FSA to increase the rate of complete removal of these lesions with a single procedure. The respective accuracy and therapeutic impact of these two techniques are compared here.

Results Intraoperative resection margin assessments were performed with FSA in 80 and SR in 35 of a total of 115 operations performed on 111 patients with pTis, pT1, or pT2 nonpalpable breast cancer. FSA diagnostic accuracy, sensitivity, and specificity were $83.8 \%, 80.0 \%$, and $87.5 \%$, respectively, compared to $60 \%, 60 \%$, and $60 \%$, respectively, for SR. FSA tended to have a stronger therapeutic impact than SR in terms of the number of patients in whom initially positive margins were rendered margin-negative thanks to intraoperative analysis and immediate reexcision or mastectomy $(27.5 \%$ vs. $14.3 \% ; p=0.124)$. More importantly, significantly fewer secondary reexcisions were performed in the FSA series than in the SR series $(12.5 \%$ vs. $37.1 \% ; p=0.002)$. Finally, the intraoperative detection of invasive cancer with FSA led to a significantly lower number of secondary procedures for axillary lymph node staging (5\% vs. $25.7 \% ; p=0.001)$.

Conclusions The present results suggest that FSA may be more accurate than SR for analyzing intraoperative resection margins during breast-conserving surgery for nonpalpable lesions.

\section{Introduction}

Large prospective randomized trials have shown that breast-conserving surgery (BCS) followed by irradiation is equally as effective as radical mastectomy when treating breast cancer [1-5]. Widespread mammographic screening 
and the concomitant detection of a growing proportion of early invasive breast cancer and ductal carcinoma in situ (DCIS) have led to a higher percentage of patients qualifying for BCS [6, 7]. Various stereotactically and sonographically guided biopsy techniques have been established for such breast lesions, which are frequently nonpalpable [8]. Although surgical margin analysis has been acknowledged to be a critical component of BCS, the best assessment technique has yet to be determined.

Historically, specimen radiography (SR) has been used to confirm that a nonpalpable radiopaque breast lesion has been removed, and resection margin status has been determined solely on the basis of permanent section analysis [9-11]. In the meantime, SR has become an accepted strategy for evaluating the completeness of resection of nonpalpable breast lesions, particularly DCIS [12-14]. The use of frozen section analysis (FSA) has not been recommended because of several limitations [15]. Nonetheless, the few published studies that have addressed the accuracy of FSA assessments of breast cancer specimen margins have reported encouraging results [16-19]. These studies, however, have not focused on nonpalpable breast lesions.

At Basel University Hospital, breast cancer surgery is performed in the gynecology and the surgery departments. One department preferably uses SR and the other uses FSA to increase the rate of complete removal of lesions involving DCIS or atypical ductal hyperplasia in a single procedure. In the present study, BCS performed on patients with nonpalpable breast lesions was evaluated retrospectively to test the hypothesis that FSA yields better results than SR in terms of accuracy and therapeutic impact for the intraoperative assessment of resection margin status.

\section{Materials and methods}

\section{Patients}

A retrospective medical chart review was performed on patients treated for nonpalpable breast lesions at Basel University Hospital between January 1990 and December 2004. Only women whose intended treatment was BCS were included. Patients and procedures in which the lumpectomy was monitored for completeness of excision solely by permanent section analysis were excluded. The following variables were recorded in an Excel file (Microsoft, Redmond, WA, USA): patient demographics; methods used to diagnose and localize the lesion for excision; therapeutic procedures in detail; and clinical and pathological features among others. The 6th edition of the American Joint Committee on Cancer (AJCC) cancer staging system was used for staging [20].
Surgical therapy and resection margin analysis

Breast surgery was performed in two departments at the Basel University Hospital Breast Center. In most cases where the preoperative biopsy detected invasive cancer only, both departments used FSA for intraoperative margin assessment. If the preoperative biopsy revealed DCIS or atypical ductal hyperplasia or if the only preoperative information available was a cytologic analysis, however, in one department the margin status was evaluated intraoperatively with SR and in the other with FSA. All mammograms were routinely discussed with the radiologist before surgery and kept at hand in the operating room until they were sent to the radiology department together with the breast specimen. During surgery there was a close collaboration between the surgical team, radiologists, and pathologists.

\section{Specimen radiography}

Specimen radiography consisted in full-field digital mammography performed with a Hologic "Selenia" imager. Most commonly, manual exposures were obtained with a molybdanium filter at exposure settings of $20 \mathrm{kV}$ and 25 mAs. Images were routinely obtained at a magnification of $1.8 \times$. At least two orthogonal planes were imaged. SR images were then compared to the preoperative mammography results.

In the one lesion that was not visible on the mammogram, sonography alone was used for initial detection, wire localization, and specimen radiography. Sonograms were obtained with a Philips HDI 5000 system fitted with a 12$\mathrm{mHz}$ linear transducer.

When the resection margin found by SR or sonogram imaging was $<1 \mathrm{~cm}$ wide, the specimen was classified as positive and reexcision was recommended.

Frozen section analysis

Frozen section analysis was performed depending on the gross pathology examination results and the pathologist's reply to the surgeon's question. Surgeons routinely oriented the breast tissue with sutures and sent the specimen intact to the Pathology Department. Multicolor inking was used to mark the surfaces of the specimen. If a tumor mass was palpable ex vivo, the specimen was sliced in 3- to 5$\mathrm{mm}$ sections perpendicular to the long axis. Margins were grossly examined for proximity to the tumor, and frozen sections were prepared of the ones located closest to the cancerous tissue for examination. Where there was no palpable ex vivo tumor and no grossly visible mass lesion, FSA was performed on breast tissue shaved circumferentially off the lumpectomy cavity or, if technically feasible, 
on all the resection margins. Margins with tumors within a 1-mm band were regarded as positive.

If the radiologist or pathologist reported that the resection was incomplete, reexcisions and intraoperative margin analyses were immediately performed until negative margins could be obtained via BCS or mastectomy (if the latter had been discussed with the patient preoperatively).

Permanent section analysis

All frozen sections were also examined in permanent paraffin-embedded sections for quality control and assurance. In all cases, additional permanent sections of the specimen were analyzed. When microcalcifications were present, permanent sections of the entire specimen were usually prepared and evaluated. A negative surgical margin was defined to be a margin free of tumor cells or atypical ductal hyperplasia for $\geq 1 \mathrm{~mm}$, whereas a positive margin was defined as containing tumor within $1 \mathrm{~mm}$.

Axillary lymph node staging was included in the primary operation protocol for all patients with breast lesions containing invasive cancer and some patients with a large DCIS. If FSA identified a previously undetected invasive component, axillary staging was performed immediately or subsequently depending on the existence of the patient's informed consent. The sentinel lymph node procedure for axillary staging was introduced at Basel University Hospital in 1997. Prior to that year, and in case of positive sentinel lymph nodes, patients were treated by level I and II axillary lymph node dissection.

\section{Interdisciplinary commissions}

All patients treated for breast cancer were discussed at interdisciplinary meetings. When permanent section analysis determined surgical margins to be positive, secondary reexcision or mastectomy was recommended. When a previously unidentified invasive component was detected, the possibility of further surgery for axillary staging was considered. Finally, specific adjuvant therapy and a followup strategy were designed for each patient at these meetings, in keeping with existing guidelines.

\section{Statistical analysis}

The chi-squared test was used to compare categorical variables, and the Mann-Whitney U-test was used to compare continuous variables. All tests were two-tailed. The significance level was defined as $p<0.05$. The tests were run with SPSS Version 13.0 for Macintosh (SPSS, Chicago, IL, USA). Diagnostic accuracy, sensitivity, specificity, and the negative and positive predictive values were calculated as described elsewhere [21].

\section{Results}

The study population consisted of 115 nonpalpable, histologically or cytologically malignant breast lesions in 111 women in whom BCS was attempted. In 80 procedures, surgical resection margin status was determined intraoperatively via FSA; in 35, the intraoperative margin was assessed with SR. In one procedure SR was performed with US techniques alone (i.e., without mammography) because the lesion was mammographically imperceptible. Patients' baseline characteristics as determined by intraoperative resection margin analysis are given in Table 1.

Final histopathology, treatment, and follow-up

The final histopathologic findings, respective tumor stages, treatment, and follow-up are given in Table 2. As expected, none of the nonpalpable breast lesions exceeded primary tumor stage pT2. In all, 19 cases of invasive cancer not histologically confirmed prior to the operation were detected by FSA.

A full long-term follow-up data set was built for 103 of these 115 procedures $(89.6 \%$ ) (Table 2$)$. Of the six patients with recurrent lesions, two had had a positive resection margin after the first excision.

Accuracy and therapeutic impact of FSA and SR

The results of intraoperative margin analysis and the respective accuracy rates after the first operation are shown by analytic technique in Table 3 . The accuracy rates were consistently higher for FSA than SR. More importantly, there was a statistical trend toward a stronger therapeutic impact of FSA than SR: $27.5 \%$ of patients in the FSA series were rendered margin-negative by intraoperative analysis and immediate reexcision or mastectomy compared to only $14.3 \%$ in the SR series ( $p=0.124)$. This clinically relevant trend was even more pronounced in the subgroup of procedures performed for DCIS or atypical ductal hyperplasia (29.8\% vs. $12.9 \%$; $p=0.083$ ). FSA was performed in 33 of the 37 operations involving lesions whose preoperative biopsy indicated invasive cancer only. In this group, the diagnostic accuracy, sensitivity, and specificity of FSA were $81.8 \%, 75.0 \%$, and $85.7 \%$, respectively; and 8 patients $(24.2 \%)$ with initially positive margins were successfully rendered margin-negative during the primary operation.

Table 4 shows the main findings of the present study. In the FSA series, axillary staging was performed significantly less frequently with a secondary procedure. More importantly, FSA yielded significantly lower rates of secondary reexcisions than SR $(12.5 \%$ vs. $37.1 \%, p=0.002)$. 
Table 1 Baseline characteristics for 115 nonpalpable breast lesions, by intraoperative margin assessment technique (FSA vs. SR)

\begin{tabular}{|c|c|c|c|c|c|}
\hline \multirow[b]{2}{*}{ Characteristic: } & \multicolumn{2}{|c|}{ Frozen section analysis } & \multicolumn{2}{|c|}{ Specimen radiography } & \multirow[t]{2}{*}{$p$} \\
\hline & No. & $\%$ & No. & $\%$ & \\
\hline Procedures & 80 & 100 & 35 & 100 & \\
\hline Patients & \multicolumn{2}{|l|}{78} & \multicolumn{2}{|l|}{33} & \\
\hline Age (years), median, range & \multicolumn{2}{|c|}{$59.6(33.9-86.4)$} & \multicolumn{2}{|c|}{$57.5(42.8-80.0)$} & 0.453 \\
\hline Mammographic lesions $^{\mathrm{a}}$ & 74 & 92.5 & 34 & 97.1 & 0.328 \\
\hline Microcalcifications & 48 & 60.0 & 28 & 80.0 & \\
\hline Nodular masses & 11 & 13.8 & 3 & 8.6 & \\
\hline Other & 14 & 17.5 & 3 & 8.6 & \\
\hline Missing data & 1 & 1.3 & 0 & 0 & \\
\hline Sonographic lesions ${ }^{\mathrm{b}}$ & 6 & 7.5 & 1 & 2.9 & \\
\hline Preoperative histology & 63 & 78.8 & 30 & 85.7 & $<0.001$ \\
\hline Invasive carcinoma & 33 & 41.3 & 4 & 11.4 & \\
\hline DCIS or ADH & 21 & 26.3 & 25 & 71.4 & \\
\hline Invasive carcinoma + DCIS or ADH & 9 & 11.3 & 1 & 2.9 & \\
\hline Malignant cytology & 17 & 21.3 & 5 & 14.3 & \\
\hline
\end{tabular}

DCIS: ductal carcinoma in situ; ADH: atypical ductal hyperplasia

${ }^{a}$ Lesions detected by mammography with or without sonography

b Lesions detected by sonography alone

\section{Discussion}

The use of FSA in connection with the complete removal of nonpalpable breast lesions in a single procedure is a matter of debate. To the authors' knowledge, this is the first study to focus on the accuracy and therapeutic impact of FSA versus intraoperative SR in this setting. The current findings suggest that FSA is a better tool than SR for reducing the need for secondary operations. Although the tendency for FSA to have a stronger therapeutic impact than SR was not statistically significant due to the small sample size, this trend is highly relevant from a clinical standpoint. Significantly fewer secondary reexcisions were performed in the FSA series than in the SR series. However, this was due partially to the fact that 12 of 33 patients $(36.4 \%)$ with positive margins after the primary operation underwent no further local surgery to obtain margin-negative tissue (9 in the FSA group, 3 in the SR group). Two patients refused any further surgery; and in two, whose pectoral fascia had been excised, the positive margin was oriented toward the pectoral muscle. In the remaining 8 cases (all elderly women who were actively involved in the decision-making process), the interdisciplinary consensus was that systematic adjuvant treatment alone would suffice, thereby sparing these patients further surgery. Finally, significantly fewer secondary axillary lymph node staging procedures were needed when FSA was used. This could be attributed, in part, to the intraoperative diagnosis of 19 invasive cancers that went undetected in the preoperative biopsy; in these cases, axillary staging could be directly undertaken during the primary procedure. Both departments preferably used FSA in cases where the preoperative biopsy detected invasive cancer only, resulting in differing volumes between the FSA and the SR series ( 80 vs. 35 procedures).

Few other retrospective studies have evaluated the role of FSA in intraoperative margin assessment in BCS patients [16-18]. In one study, SR was used for most of the nonpalpable lesions, and FSA was preferably applied for palpable breast masses [16]. Of a total population of 264 patients, $29 \%$ with invasive cancer but only $9 \%$ of those with DCIS who had initial positive/close margins were rendered margin-negative by intraoperative analysis and immediate reexcision. The present study yielded similar numbers, although the aforementioned difference between invasive cancer and DCIS was not observed. From a clinical perspective, the type of carcinoma (invasive versus DCIS) ultimately detected by permanent section analysis is of no relevance to the surgeon's decision about which intraoperative analytic technique to use to determine margin status. By contrast, DCIS detected by the preoperative biopsy is highly relevant to the surgeon. The present study showed that, thanks to FSA, in 14 of the 47 instances (29.8\%) in which cytologic testing or a preoperative biopsy was indicative of DCIS, a single operation sufficed to render initially margin-positive patients margin-negative. Despite the focus on nonpalpable breast lesions in the present study, specificity, sensitivity, and diagnostic accuracy of FSA were almost identical in the Cabioglu et al. study [16] and the present study $(9.17 \%, 77.8 \%$, and 
Table 2 Histopathological findings, tumor stages, treatment, and follow-up of 115 nonpalpable breast lesions by intraoperative margin assessment techniques

\begin{tabular}{|c|c|c|c|c|c|}
\hline \multirow[t]{2}{*}{ Parameter } & \multicolumn{2}{|c|}{ Frozen section analysis } & \multicolumn{2}{|c|}{ Specimen radiography } & \multirow[t]{2}{*}{$p$} \\
\hline & No. & $\%$ & No. & $\%$ & \\
\hline Procedures (no.) & 80 & 100 & 35 & 100 & \\
\hline \multicolumn{6}{|l|}{ Histologic results of FSA } \\
\hline Invasive carcinoma & 47 & 58.8 & $\mathrm{n} / \mathrm{a}$ & & \\
\hline DCIS & 13 & 16.3 & $\mathrm{n} / \mathrm{a}$ & & \\
\hline Invasive carcinoma + DCIS $^{a}$ & 14 & 17.5 & $\mathrm{n} / \mathrm{a}$ & & \\
\hline Other & 6 & 7.5 & $\mathrm{n} / \mathrm{a}$ & & \\
\hline \multicolumn{6}{|l|}{ Final histologic results } \\
\hline Invasive carcinoma & 31 & 38.8 & 5 & 14.3 & \\
\hline $\operatorname{DCIS}^{a}$ & 12 & 15 & 21 & 60 & \\
\hline Invasive carcinoma + DCIS $^{a}$ & 37 & 46.3 & 9 & 25.7 & $<0.001$ \\
\hline \multicolumn{6}{|l|}{ Borderline lesions } \\
\hline Atypical ductal hyperplasia & 6 & 7.5 & 4 & 11.4 & \\
\hline Atypical lobular hyperplasia & 4 & 5 & 3 & 8.6 & \\
\hline Lobular carcinoma in situ & 12 & 15 & 1 & 2.9 & 0.242 \\
\hline \multicolumn{6}{|l|}{ TNM staging } \\
\hline \multicolumn{6}{|l|}{ Primary tumor $(\mathrm{T})$} \\
\hline Tis & 12 & 15 & 21 & 60 & \\
\hline $\mathrm{T} 1$ & 60 & 75 & 14 & 40 & \\
\hline $\mathrm{T} 2$ & 8 & 10 & 0 & 0 & $<0.001$ \\
\hline \multicolumn{6}{|l|}{ Regional lymph nodes (N) } \\
\hline $\mathrm{cNO}$ & 11 & 13.8 & 20 & 57.1 & \\
\hline $\mathrm{pNO}$ & 35 & 43.8 & 12 & 34.3 & \\
\hline $\mathrm{pN} 0(\mathrm{sn})$ & 18 & 22.5 & 3 & 8.6 & \\
\hline $\mathrm{pN} 1 \mathrm{mi}$ & 4 & 5 & 0 & 0 & \\
\hline $\mathrm{pN} 1$ & 9 & 11.3 & 0 & 0 & \\
\hline $\mathrm{pN} 2$ & 2 & 2.5 & 0 & 0 & \\
\hline $\mathrm{pN} 3$ & 1 & 1.3 & 0 & 0 & $<0.001$ \\
\hline \multicolumn{6}{|l|}{ Distant metastases (M) } \\
\hline M0 & 80 & 100 & 35 & 100 & \\
\hline M1 & 0 & 0 & 0 & 0 & 1.000 \\
\hline \multicolumn{6}{|l|}{ Stage according to AJCC } \\
\hline 0 & 12 & 15 & 21 & 60 & \\
\hline I & 50 & 62.5 & 14 & 40 & \\
\hline IIA & 9 & 11.3 & 0 & 0 & \\
\hline IIB & 6 & 7.5 & 0 & 0 & \\
\hline IIIA & 2 & 2.5 & 0 & 0 & \\
\hline IIIB & 0 & 0 & 0 & 0 & \\
\hline IIIC & 1 & 1.3 & 0 & 0 & $<0.001$ \\
\hline \multicolumn{6}{|l|}{ Primary operation } \\
\hline BCT & 70 & 87.5 & 35 & 100 & \\
\hline Mastectomy & 10 & 12.5 & 0 & 0 & 0.029 \\
\hline \multicolumn{6}{|l|}{ Primary axillary lymph node staging } \\
\hline Axillary lymph node dissection ${ }^{a}$ & 47 & 58.8 & 3 & 8.6 & \\
\hline Sentinel lymph node dissection alone & 18 & 22.5 & 3 & 8.6 & \\
\hline No axillary lymph node staging & 15 & 18.8 & 29 & 82.9 & $<0.001$ \\
\hline
\end{tabular}


Table 2 continued

\begin{tabular}{|c|c|c|c|c|c|}
\hline \multirow[t]{2}{*}{ Parameter } & \multicolumn{2}{|c|}{ Frozen section analysis } & \multicolumn{2}{|c|}{ Specimen radiography } & \multirow[t]{2}{*}{$p$} \\
\hline & No. & $\%$ & No. & $\%$ & \\
\hline Complete long-term follow-up data sets & 75 & 93.8 & 28 & 80.0 & \\
\hline Follow-up (years), median, range & \multicolumn{2}{|c|}{$4.4(0.1-14.1)$} & \multicolumn{2}{|c|}{$3.9(0.3-8.0)$} & 0.258 \\
\hline Recurrences & 4 & 5.0 & 2 & 5.7 & 0.874 \\
\hline Deaths & 6 & 7.5 & 0 & 0 & 0.096 \\
\hline
\end{tabular}

n/a: not applicable; AJCC: American Joint Committee on Cancer; BCT: breast-conserving therapy

${ }^{a}$ With or without sentinel lymph node dissection

Table 3 Intraoperative margin assessment results, therapeutic impact, and accuracy rates after the first operation, by assessment technique (FSA vs. SR)

\begin{tabular}{|c|c|c|c|c|c|c|c|c|c|c|c|c|c|c|c|c|}
\hline \multirow{4}{*}{ Parameter } & \multicolumn{8}{|c|}{ Total study population (115 procedures) } & \multicolumn{8}{|c|}{ DCIS or ADH in preoperative biopsy $(n=78)$} \\
\hline & \multicolumn{4}{|c|}{$\begin{array}{l}\text { Margin with FSA } \\
(n=80)\end{array}$} & \multicolumn{4}{|c|}{$\begin{array}{l}\text { Margin with SR } \\
(n=35)\end{array}$} & \multicolumn{4}{|c|}{$\begin{array}{l}\text { Margin with FSA } \\
(n=47)\end{array}$} & \multicolumn{4}{|c|}{$\begin{array}{l}\text { Margin with SR } \\
(n=31)\end{array}$} \\
\hline & \multicolumn{2}{|c|}{ Negative $^{a}$} & \multicolumn{2}{|c|}{ Positive $^{\mathrm{b}}$} & \multicolumn{2}{|c|}{ Negative $^{\mathrm{a}}$} & \multicolumn{2}{|c|}{ Positive $^{\mathrm{b}}$} & \multicolumn{2}{|c|}{ Negative $^{\mathrm{a}}$} & \multicolumn{2}{|c|}{ Positive $^{\mathrm{b}}$} & \multicolumn{2}{|c|}{ Negative $^{\mathrm{a}}$} & \multicolumn{2}{|c|}{ Positive $^{\mathrm{b}}$} \\
\hline & No. & $\%$ & No. & $\%$ & No. & $\%$ & No. & $\%$ & No. & $\%$ & No. & $\%$ & No. & $\%$ & No. & $\%$ \\
\hline Intraoperative diagnosis by FSA or SR & 43 & 53.8 & 37 & 46.3 & 17 & 48.6 & 18 & 51.4 & 22 & 46.8 & 25 & 53.2 & 15 & 48.4 & 16 & 51.6 \\
\hline Diagnosis by permanent section analysis & 35 & 43.8 & 32 & 40.0 & 9 & 25.7 & 12 & 34.3 & 17 & 36.2 & 23 & 48.9 & 7 & 22.6 & 10 & 32.3 \\
\hline Positive margin turned into negative & 0 & 0 & 22 & 27.5 & 0 & 0 & 5 & 14.3 & 0 & 0 & 14 & 29.8 & 0 & 0 & 4 & 12.9 \\
\hline $\begin{array}{l}\text { Positive margin at the end of primary } \\
\text { operation }\end{array}$ & 8 & 10 & 10 & 12.5 & 8 & 22.9 & 7 & 20.0 & 5 & 10.6 & 9 & 19.1 & 8 & 25.8 & 6 & 19.4 \\
\hline Diagnostic accuracy (\%) & 83.8 & & & & 60.0 & & & & 85.1 & & & & 54.8 & & & \\
\hline Sensitivity (\%) & 80.0 & & & & 60.0 & & & & 82.1 & & & & 55.6 & & & \\
\hline Specificity (\%) & 87.5 & & & & 60.0 & & & & 89.5 & & & & 53.8 & & & \\
\hline Negative predictive value (\%) & 81.4 & & & & 52.9 & & & & 77.3 & & & & 46.7 & & & \\
\hline Positive predictive value $(\%)$ & 86.5 & & & & 66.7 & & & & 92.0 & & & & 62.5 & & & \\
\hline
\end{tabular}

FSA: frozen section analysis; SR: specimen radiography

${ }^{a}$ Margin $\geq 1 \mathrm{~mm}$ free of tumor

b Margin involving tumor or $<1 \mathrm{~mm}$ free of tumor

Table 4 Secondary operations performed in the FSAand SR series

\begin{tabular}{|c|c|c|c|c|c|}
\hline \multirow[t]{2}{*}{ Parameter } & \multicolumn{2}{|c|}{ Frozen section analysis } & \multicolumn{2}{|c|}{ Specimen radiography } & \multirow[t]{2}{*}{$p$} \\
\hline & No. & $\%$ & No. & $\%$ & \\
\hline Procedures (no.) & 80 & 100 & 35 & 100 & \\
\hline Frequency of secondary reexcision & $10 / 80$ & 12.5 & $13 / 35$ & 37.1 & 0.002 \\
\hline \multicolumn{6}{|l|}{ Type of secondary operation } \\
\hline BCT & 3 & 3.8 & 8 & 22.9 & \\
\hline Mastectomy & 7 & 8.8 & 5 & 14.3 & 0.133 \\
\hline Frequency of secondary axillary lymph node staging & $4 / 80$ & 5.0 & $9 / 35$ & 25.7 & 0.001 \\
\hline
\end{tabular}

$87.4 \%$, respectively, compared to $87.5 \%, 80.0 \%$, and $83.8 \%$, respectively). Moreover, in the present study the FSA accuracy rates were similar $(89.5 \%, 82.1 \%$, and $85.1 \%$, respectively) for the subgroup of procedures in which DCIS or atypical ductal hyperplasia was suspected based on preoperative histocytologic analysis. Another fact of interest is that of 52 patients in the above study [16] for whom permanent section analysis showed final positive/ close margins only $23(44.2 \%)$ were subjected to a second procedure to obtain wider negative margins, compared to 
21 of the $33(63.6 \%)$ such patients in the study reported here. This is relevant inasmuch as it shows that not all patients with positive/close margins after a first tumoral excision undergo subsequent local reexcision.

Two other studies support the present findings to the effect that FSA may be beneficial in most instances of BCS $[17,18]$. In one [18], the margins of specimens excised from 97 BSC patients were evaluated using FSA. A total of $59 \%$ of the excisions were needle-localization guided, whereas the diagnostic accuracy of FSA came to $84 \%$. The authors recommended FSA for BCS in general but concluded that DCIS was more difficult to identify with FSA given the significantly higher rates of false negatives found in this group of patients. Finally, Camp et al. [17] retrospectively reviewed BCS in 220 patients with T1/2 early invasive breast cancer and 47 patients with DCIS using margins shaved intraoperatively for FSA. They concluded that FSA could lower reexcision rates in similar proportions in the two groups, but they did not distinguish between palpable and nonpalpable lesions.

The eight false negatives returned by FSA merit some comment. Freezing artifacts, a well known limitation of FSA, may have been responsible in a few cases. Another possible source, however, may have been sampling errors in large specimens for which only a small number of gross examination and surgeon references were available to the pathologist. Sampling errors should be minimized by cooperating closely with the pathologist, who should be allowed, wherever possible, to focus on the margin closest to the tumor.

The limitations of this study are acknowledged. Most relevantly, as this is a retrospective study based on a review of medical charts rather than a prospective randomized trial, the two series of patients differed in terms of a number of baseline variables. For instance, FSA was used significantly more often than SR for invasive lesions identified by preoperative biopsy/cytology, and significant differences were observed between the tumor stages recorded in the two series.

Second, partly due to the application of strict exclusion criteria, this study covered only a small number of procedures. Third, over the fairly long period studied, surgeons may have modified the lumpectomy technique used in an attempt to minimize secondary reexcisions and locoregional recurrence while maintaining suitable cosmesis. Nonetheless, of the total 115 procedures, only 19 were performed during the first half of the period. This distribution may suggest the increasing effectiveness of screening programs in detecting nonpalpable breast lesions over the period studied or the growing use of intraoperative margin assessment to treat these lesions; in any event, the results reported here seem to reflect current surgical practice at Basel University Hospital.

\section{Conclusions}

Summarizing, FSA seems to be more accurate than SR for intraoperative margin assessment during BCS for nonpalpable breast lesions, regardless of the histopathologic nature of the lesion. FSA significantly reduces the number of secondary reexcisions and secondary axillary lymph node staging procedures. Due to the retrospective nature and the small power of the evidence presented, these findings must be corroborated by larger-scale prospective studies.

\section{References}

1. Arriagada R, Le MG, Rochard F et al (1996) Conservative treatment versus mastectomy in early breast cancer: patterns of failure with 15 years of follow-up data; Institut Gustave-Roussy Breast Cancer Group. J Clin Oncol 14:1558-1564

2. Fisher B, Redmond C, Poisson R et al (1989) Eight-year results of a randomized clinical trial comparing total mastectomy and lumpectomy with or without irradiation in the treatment of breast cancer. N Engl J Med 320:822-828

3. Fisher B, Anderson S, Bryant J et al (2002) Twenty-year followup of a randomized trial comparing total mastectomy, lumpectomy, and lumpectomy plus irradiation for the treatment of invasive breast cancer. N Engl J Med 347:1233-1241

4. Jacobson JA, Danforth DN, Cowan KH et al (1995) Ten-year results of a comparison of conservation with mastectomy in the treatment of stage I and II breast cancer. N Engl J Med 332: 907-911

5. Veronesi U, Banfi A, Salvadori B et al (1990) Breast conservation is the treatment of choice in small breast cancer: long-term results of a randomized trial. Eur J Cancer 26:668-670

6. Ernster VL, Barclay J, Kerlikowske K et al (1996) Incidence of and treatment for ductal carcinoma in situ of the breast. JAMA 275:913-918

7. Ernster VL, Ballard-Barbash R, Barlow WE et al (2002) Detection of ductal carcinoma in situ in women undergoing screening mammography. J Natl Cancer Inst 94:1546-1554

8. Weber WP, Zanetti R, Langer I et al (2005) Mammotome: less invasive than ABBI with similar accuracy for early breast cancer detection. World J Surg 29:495-499

9. Letton AH, Mason EM (1980) The treatment of nonpalpable carcinoma of the breast. Cancer 46:980-982

10. Rosen PP (1991) Pathological assessment of nonpalpable breast lesions. Semin Surg Oncol 7:257-260

11. Snyder RE (1980) Specimen radiography and preoperative localization of nonpalpable breast cancer. Cancer 46:950-956

12. Graham RA, Homer MJ, Sigler CJ et al (1994) The efficacy of specimen radiography in evaluating the surgical margins of impalpable breast carcinoma. AJR Am J Roentgenol 162:33-36

13. Mazouni C, Rouzier R, Balleyguier C et al (2006) Specimen radiography as predictor of resection margin status in non-palpable breast lesions. Clin Radiol 61:789-796

14. Sakorafas GH, Farley DR (2003) Optimal management of ductal carcinoma in situ of the breast. Surg Oncol 12:221-240

15. Klimberg VS, Harms S, Korourian S (1999) Assessing margin status. Surg Oncol 8:77-84

16. Cabioglu N, Hunt KK, Sahin AA et al (2007) Role for intraoperative margin assessment in patients undergoing breastconserving surgery. Ann Surg Oncol 14:1458-1471 
17. Camp ER, McAuliffe PF, Gilroy JS et al (2005) Minimizing local recurrence after breast conserving therapy using intraoperative shaved margins to determine pathologic tumor clearance. J Am Coll Surg 201:855-861

18. Cendan JC, Coco D, Copeland EMIII (2005) Accuracy of intraoperative frozen-section analysis of breast cancer lumpectomybed margins. J Am Coll Surg 201:194-198
19. Weber S, Storm FK, Stitt J et al (1997) The role of frozen section analysis of margins during breast conservation surgery. Cancer $\mathrm{J}$ Sci Am 3:273-277

20. Greene FL, Page DL, Fleming ID (2002) AJCC Cancer staging manual, 6th edn. Springer, New York, p 223

21. Walter SD (1998) Sensitivity. In: Armitage P, Colton T (eds) Encyclopedia of biostatistics. Toronto, Wiley, pp 4053-4054 